\title{
La participación de la escuela de Brentano en el \\ Psycologismusstreit
}

\author{
The participation of the Brentano school in the \\ Psycologismusstreit
}

MÁRIO ARIEl GONZÁLEZ PORTA a

\section{Resumen}

El tema de la participación de la escuela de Brentano en la polémica en torno al psicologismo no se deja reducir a una simple y única pregunta, ni se puede responder con una simple y única respuesta, pues presenta una marcada diversidad tanto desde el punto de vista sistemático como histórico, componiéndose de diferentes polémicas que tienen un cierto grado de independencia.

Palabras clave: Brentano. Stumpf. Marty. Psicologismo. Método psicológico.

\section{Abstract}

The theme of the participation of the Brentano school in the controversy surrounding psychologism does not allow himself to be reduced to a single and unique question, nor can he respond with a single and unique answer, since he presents a marked diversity both from a systematic and historical point of view, consisting of different polemics that have a certain degree of independence.

Keywords: Brentano. Stumpf. Marty. Psychologism. Psychological method.

a Pontifícia Universidade Católica de São Paulo (PUC-SP), São Paulo, Brasil. Doutor em Filosofia, e-mail: mariopor@pucsp.br 


\section{Introducción}

El tema del psicologismo en la escuela de Brentano y la participación de ésta en la polémica respectiva, es algo que aún espera por un tratamiento adecuado. Al día de hoy, el status questionis podría ser resumida en dos puntos:

a. si bien existen varios estudios que se ocupan con el problema del psicologismo en la escuela de Brentano (Heidegger (1914), Kastil (1951), Cernoch (1980), Rollinger (1999, 2004), Modenato (1995), Cavallin (1997), Jaquette (2004), Huemer (2004), Frechette (2016), lo hacen siempre desde perspectivas parciales y con intereses puntuales, permaneciendo en definitiva inconexos entre sí. Falta, pues, una visión de conjunto que unifique esas perspectivas y, de este modo, permita dimensionarlas e interrelacionarlas adecuadamente;

b. el libro standard sobre el Psicologismusstreit (PS) de autoría de Martin Kusch, tiene como una de sus grandes carencias abordar la escuela de Brentano de modo insuficiente e inadecuado, habiendo sido por tal motivo duramente criticada por Kevin Mulligan en su reseña (2009).

Esta situación no es casual y merece una reflexión. La forma en que el libro de Kusch tematiza la escuela de Brentano, no debe ser meramente constatada, sino comprendida. Si, en la reconstrucción del PS en torno a los "Prolegómenos", el libro de Kusch es insuperable e inició el estudio detenido de materiales que sólo se citaban aleatoriamente, sus opciones previas implícitas terminan condicionando y comprometiendo sus resultados. En efecto, Kusch toma Husserl como punto de referencia y ordena la discusión histórica y sistemáticamente a partir de él. Mas los "Prolegómenos", y pese a su importancia decisiva, deben ser vistos como más un momento del PS y, de este modo, colocados ellos mismos en perspectiva, en vez de ser ellos la perspectiva a partir de la cual se considera la totalidad. Si bien no cabe duda que los "Prolegómenos" proporcionan una importante ordenación del PS, dado su propio objetivo, ellos no están preocupados en distinguir, sino que tienden a nivelar, tratando "el" psicologismo como si fuera una unidad. Los problemas de tal modo de proceder terminan repercutiendo en el tratamiento de la escuela de Brentano, pues Kusch no meramente de hecho aborda la escuela de Brentano de un modo tangencial e insuficiente, sino que, más aun, no dispone de los recursos hermenéuticos necesarios para hacerlo satisfactoriamente. 


\section{Algunas distinciones conceptuales básicas necesarias}

La clarificación de la participación de Brentano y su escuela en el PS exige el establecimiento de varias distinciones.

a. Debemos distinguir con claridad dos problemas diferentes, aun cuando interrelacionados, a saber, el problema del método psicológico (MP) y el problema del método de la psicología. Para alcanzar tal objetivo, es imprescindible una consideración terminológica. La expresión "MP" tiende a ser usada desde fines del siglo XIX y comienzos del XX en el sentido de "método de la psicología”, o sea, para referirse a la cuestión de cual deba ser el modo de proceder de una cierta disciplina para constituirse como ciencia autónoma. En mediados del siglo XIX, sin embargo, dicha expresión tiene un otro sentido usual referente a cuál deba ser el método de la filosofía, cuestión ésta generalmente colocada en el horizonte de una caracterización de la filosofía como ciencia que asegure su derecho a existencia en la llamada “crisis de identidad”. La idea básica es qué si toda ciencia debe fundarse en la experiencia y la ciencia de la naturaleza (Naturwissenschaft) trata de la experiencia externa, corresponde a la filosofía fundarse en la experiencia interna. En este sentido, la expresión "MP" fue originariamente el opuesto, primero, del método especulativo propio del idealismo alemán, después, del método transcendental neokantiano y, finalmente, del método fenomenológico. Si, como es sabido, la idea del MP hunde sus raíces en el empirismo inglés, ella se constituye en la filosofía alemana como alternativa de perfil especifico a partir de la obra de Fries y Beneke. La expresión "MP”, sin embargo, fue solo introducida décadas después por Bona Meyer y popularizada por sus oponentes neokantianos.

b. Si tenemos en cuenta la distinción entre MP y método de la psicología, entonces podemos ulteriormente distinguir entre MP y psicologismo. Entenderemos por "psicologismo" una tesis o teoría filosófica que reduce entidades de diferentes tipos a entidades psicológicas, siendo que, en tal reducción, generalmente se 
implica una consecuencia relativista. Entenderemos por MP, por el contrario, una postura puramente metodológica, en principio neutra con respecto a cualquier tesis o teoría filosófica específica. La distinción entre psicologismo y MP permite colocar explícitamente dos cuestiones discutidas intensamente en el siglo XIX, referentes, por un lado, a las posibilidades fundacionales del MP, por otro, a si el MP conducía necesariamente o no al psicologismo. En tanto que algunos defensores del MP resistieron a la identificación de MP y psicologismo, los autoproclamados anti-psicologistas tendieron a presuponer sin más la misma. En base a lo dicho, obsérvese que un aspecto bien definido del Psychologismusstreit giró, no en torno a una discusión entre psicologistas y anti-psicologistas, sino entre anti-psicologistas y defensores del MP.

c. Existen diferentes formas de psicologismo y diferentes criterios posibles para clasificar las mismas. Algunos, sin embargo, son ciertamente más relevantes, pues permiten evitar confusiones y establecer relaciones precisas sobre la base de distinciones relativamente simples. Tal es el caso del criterio temático que diferencia entre psicologismo lógico, epistemológico y semántico, según se trata de un psicologismo en la lógica, en la teoría del conocimiento o en la semántica. Dicho de otro modo, si toda forma de psicologismo es reduccionista, el psicologismo lógico consiste en la reducción de leyes lógicas a psicológicas, el epistemológico de procesos cognitivos a procesos psicológicos y el semántico de significaciones a representaciones (Vorstellungen). Lo importante de estas distinciones radica en que se puede ser psicologista en una esfera y no en la otra.

d. En "Prolegómenos" Husserl introduce la importante distinción entre relativismo genérico e individual (HUSSERL, 1975, p. 122ss.). Psicologismo y relativismo genérico no son sinónimos pues, aun cuando se conceda que el psicologismo como tal implica alguna forma de relativismo, la recíproca no vale, dado que existen ciertamente formas de relativismo que no son psicologistas (como sociologismo o historicismo). Psicologismo y relativismo individual, por su vez, se encuentran en variadas relaciones, siendo para ello decisivo el si se trata del 
psicologismo lógico, epistemológico o semántico. El psicologismo lógico y/o epistemológico, es en general un relativismo de la especie, no siendo en forma alguna característico del siglo XIX la idea de un relativismo lógico y/o epistemológico individual. Por el contrario, es usual en el siglo XIX, y por influencia de la tradición lockeana, un cierto relativismo individual o solipsismo en la semántica.

e. Ni psicologismo ni anti-psicologismo constituyeron partidos homogéneos, sino que dentro de cada uno de ellos pueden ser identificadas variaciones. Podemos clasificar los modos de anti-psicologismo en tres formas básicas:

- el realismo lógico (Bolzano, Frege y Husserl I), que considera que sólo se puede evitar el relativismo si se afirma la existencia de un reino platónico de objetos abstractos;

- el neokantismo (Cohen, Windelband, Riehl, Rickert, Natorp y Cassirer), qué, aun negando la existencia de objetos abstractos, considera necesario aceptar la idea de conocimiento a priori para evitar el relativismo, siendo que este conocimiento a priori se restringe a las condiciones de posibilidad del conocimiento empírico y

- la fenomenología transcendental (Husserl II), que considera que el relativismo solo es superado de modo definitivo a través de la reducción, la cual abre un ámbito de experiencia transcendental intuitivamente accesible.

- Aun cuando el anti-psicologismo del realismo lógico se puede extender a la epistemología, él se concentra primariamente en la lógica y la semántica. El anti-psicologismo neokantiano, por su vez, se focaliza en la epistemología, resistiendo la idea de una lógica que no sea transcendental y no mostrando sensibilidad alguna para la cuestión semántica. El anti-psicologismo fenomenológico-transcendental, finalmente, tiene un interés primario por la epistemología, de la crítica de cuyo psicologismo deriva, pero se proyecta con igual intensidad en la lógica y la semántica.

\section{Perspectiva histórica}

Sobre la base de las consideraciones anteriores, podemos ordenar el problema del psicologismo en la escuela de Brentano estableciendo de principio una distinción básica entre la perspectiva histórica y sistemática.

Del punto de vista histórico-cronológico, tenemos cuatro elementos importantes que deben ser colocados en primer plano: 
a. La relación de la escuela de Brentano con el psicologismo no comienza con la publicación de los "Prolegómenos" husserlianos.

b. La cuestión del psicologismo no se delinea como tema específico ni juega ningún papel en el inicio de la reflexión brentaniana. Aun cuando Brentano, en un primer momento, no tiene contacto con Bolzano, sí lo tiene con Herbart y Lotze. Sin embargo, el no parece tener sensibilidad alguna para el problema del psicologismo que ya se anuncia en éstos.

c. La evolución de la escuela de Brentano es paralela al desarrollo del PS y acompaña las diferentes etapas del mismo. Por tal razón, se debe tener clareza de cuál momento de la escuela se está considerando en cada caso particular y, correlativamente, cuál es el tipo de anti-psicologismo con respecto al cual ella debe posicionarse.

d. El problema del psicologismo y la participación en el PS comienza a ser significativo en la escuela de Brentano a partir del momento en que, mediante la acción del neokantismo, el PS se coloca como una prioridad en la agenda filosófica.

Dado lo anterior, en la relación entre la escuela de Brentano y el psicologismo se pueden diferenciar al menos cinco momentos, debiéndose prestar particular atención a cuál o cuáles miembros de la escuela juegan en cada caso el rol decisivo. En esquema tenemos:

a. polémica con el neokantianismo;

b. polémica con el realismo lógico;

c. polémica con la fenomenología transcendental;

d. reacción al Apsicologismo y

e. polémicas internas a la escuela.

\section{Perspectiva sistemática}

La escuela de Brentano ha sido objeto de crítica de parte de los anti-psicologistas. Ahora bien, para entender y juzgar adecuadamente las mismas, es esencial distinguir entre dos tipos de críticas diferentes, a saber, críticas referentes al MP en cuanto tal y críticas que se dirigen a doctrinas particulares de la escuela o de alguno de sus integrantes. Si se atiende a lo anterior, es claro que hay dos formas diferentes de colocar la pregunta por la relación de la escuela de Brentano al psicologismo. 


\section{Psicologismo y MP en la escuela de Brentano}

Con respecto a los motivos generales, es decisivo tener en claro y diferenciar tres tesis.

a. La escuela de Brentano es defensora del MP, constituyendo el mismo un elemento esencial de su autoidentidad (BRENTANO, 2013, p. 137; 1971, I, p. $30 ; 1880$, p. $67 ; 1893$, p. $14 ; 1983$, p. 29-30).

b. Ella como un todo, sin embargo, ha negado la pertinencia de ser considerada psicologista por este motivo (BRENTANO: 1971, II, p. 181-188; MARTY: 1908, p. 12; Kraus 1971, p. LXI).

c. Mas aún, ella se ha entendido a sí misma como anti-psicologista, esto es, ha defendido la idea de que a partir del MP es posible desarrollar una forma propia y eficiente de anti-psicologismo.

Para profundizar adecuadamente esas cuestiones, debemos aplicar a cada una de ellas las distinciones anteriormente efectuadas, o sea,

a. debemos distinguir, en primer lugar, la pregunta general de si el MP en cuanto tal conduce al psicologismo (lo cual, dado la diversidad de variantes, tal vez sea una pregunta sin sentido), de la pregunta de si la variante peculiar brentaniana del MP conduce al psicologismo y

b. dado que existen diferentes formas de psicologismo, la pregunta no puede meramente ser de si el MP brentaniano conduce o no al psicologismo, sino si el conduce o no al psicologismo en la lógica, en la epistemología y en la semántica.

\section{Epistemología y MP: la controversia de la escuela de Brentano con el neokantianismo}

La distinción entre análisis psicológico-empírico y análisis lógico-conceptual es esencial en el neokantismo para delimitar el método transcendental frente al psicológico. El primero tiene por objeto el proceso real del conocer (Erkennen), el segundo el contenido lógico-objetivo del mismo (Erkenntnis), plasmado en última instancia en la ciencia.

La polémica entre método transcendental y MP es una sub-polémica bien delimitada dentro del Psychologismusstreit, siendo la escuela de Brentano un participante decisivo de la misma en su forma pos-neokantiana. Un escrito típico de esta nueva situación es "Psychologie und Erkenntnistheorie" de Stumpf. Este texto es importante por dos motivos: a. porque él es el primero que, ya en 1896 (o sea, cuatro años antes de 
"Investigaciones lógicas"), sistematiza la polémica en torno al psicologismo, ordenando dialécticamente los argumentos principales de ambos partidos; b. porque él tiene en cuenta las distinciones neokantianas y, al delimitar claramente dentro del MP la línea de la psicología genético-causal de la de la psicología descriptiva, establece de un modo más diferenciado la relación psicología — teoría del conocimiento.

Decir que Brentano introdujo el concepto de intencionalidad en la filosofía alemana del s. XIX, es ciertamente una obviedad. No lo es, sin embargo, el llamar la atención sobre la importancia del mismo para una reformulación del MP. Desde su inicio, el MP era fundamentado en la oposición entre la percepción interna y externa, definiéndose por tanto lo psíquico por su modo de acceso y, de este modo, dando posibilidades limitadas a la descripción del mismo, tales como la constatación de antecedencias, consecuencias y simultaneidades temporales. Con la introducción del concepto de intencionalidad, sin embargo, lo psíquico pasa a definirse por una característica intrínseca (STUMPF, 1874, p. 207) y, con ello, la percepción interna a ser concebida como co-consciencia del acto (BRENTANO, 1971, I, p. 141 ss.), con lo cual se abren nuevas posibilidades de descripción, tales como la consideración in recto e in obliquo del objeto (BRENTANO, 1971, II, p. 142ss.). Estas novedades permiten un nuevo tipo de estudio, el estudio de génesis intencional o "análisis de origen” (STUMPF, 1874, p. 214). Una consecuencia de tal nueva situación para el PS es que muda el propio sentido de la distinción entre validad y génesis (Geltung und Genese), planteada prioritariamente por los neokantianos en vistas a una psicología orientada por el modelo metodológico de la ciencia natural, ofreciéndose nuevas posibilidades de entender la misma.

Stumpf niega el psicologismo en el sentido de una reducción de la epistemología a la psicología. Ambas disciplinas son diferentes y tienen tareas específicas; la psicología tiene que ver con el origen de conceptos, la epistemología con la verdad de enunciados. Pero, si ambas disciplinas son diferentes, esto no impide que ellas se encuentren en una interrelación, interrelación ésta que puede ser resumida en la tesis de que nada puede ser transcendentalmente verdadero y psicológicamente falso. Ahora bien, la psicología 
kantiana es falsa y, por tanto, también toda la epistemología transcendental que en ella reposa y que presupone, en última instancia, la distinción entre materia y forma del conocimiento.

El escrito de Stumpf abre en la escuela de Brentano una línea polémica contra el neokantianismo que se prolonga a través de la respuesta de Marty a la acusación de psicologismo de Adickes (MARTY, 1908, p. 6ss.; ADICKES, 1899., p. 856) y el análisis crítico de Kraus respecto de Cohen (KRAUS, 1929, 1971, p. 14).

Los aspectos principales de la comparación entre la postura neokantiana y brentaniana frente a las relaciones entre psicología y epistemología puede ser resumido en los siguientes puntos

a. Entre el neokantianismo y la escuela de Brentano existen dos ideas esencialmente diferentes de epistemología (y, en definitiva, de filosofía) que terminan repercutiendo en la diversa relación en que ambos sitúan psicología y teoría del conocimiento. Para el neokantianismo, la epistemología es un discurso de segunda orden y, por tal razón, posee una naturaleza esencialmente lógicoreflexiva. Para la escuela de Brentano, por el contrario, la teoría del conocimiento no es otra cosa que el establecimiento de aquellas verdades que son por sí mismas evidentes, estando el cumplimiento de esta tarea indisociablemente relacionado a la percepción interna y, por tanto, a la psicología.

b. La diferente concepción de teoría del conocimiento en ambos movimientos está vinculado al diferente tratamiento de la relación entre epistemología y metafísica. Para la escuela de Brentano la teoría del conocimiento remite, en última instancia, a una ontología de la subjetividad y, por tanto, no se opone a la metafísica sino que es parte de ella (MARTY, 1896, p. 79-81; ESCHENMEYER, 1914, p. 99101; STUMPF, 1924, p. 28 (232)).

\section{Lógica y MP: controversia con el realismo lógico}

Desde los comienzos de su reflexión, Brentano tiene como uno de sus objetivos fundamentales una renovación de la lógica (SIMONS, 1987; KAMITZ, 1989). Para tal renovación, la fundamentación de la lógica en la psicología juega un papel fundamental. Dado que la lógica se ocupa de la verdad y, según una vieja tradición que se remonta a Aristóteles y de la cual Brentano se hace eco, el portador de verdad es el juicio, es claro que el objeto de la lógica es el juicio. Pero, parece obvio que la correcta determinación de la naturaleza del juicio solo puede ser tarea de una psicología descriptiva. En 
consecuencia, la lógica debe basarse en los resultados de tal psicología. La enseñanza fundamental que nos deja una correcta descripción del acto de juzgar es que él es diferente de un acto de mero representar y que se efectúa sobre él, el cual le proporciona su objeto, mediante una afirmación o negación. Del hecho psicológico de que todo acto de juzgar consiste en afirmar o negar, Brentano deriva la tesis lógica de que, en última instancia, todo juicio es existencial y, de ésta, la necesidad de una reformulación de la silogística aristotélica.

Para el Brentano de madurez, el fundamento último de la verdad es la evidencia. Ahora bien, la evidencia no siempre es inmediata, sino que muchas veces es mediada por otras. Existen, pues, dos tipos de verdades, las evidentes y las no-evidentes, siendo que las últimas tienen que fundarse en las primeras. La lógica nada mas es que el estudio de los modos y formas de fundamentación mediata de la verdad, o sea, una guía para el juicio correcto y, por tal razón, un arte o Kunst (BRENTANO, 1880, p. 3dd.). Uno de estos modos, aun cuando ciertamente no el único (pues la lógica contiene también como momento esencial una teoría del simbolismo), es la inferencia. El desarrollo de una teoría de la inferencia conduce al establecimiento de leyes lógicas y, en última instancia, de principios indemostrables, de los cuales el fundamental es el de identidad y nocontradicción. Estas leyes y estos principios dicen algo respecto del juzgar, por ejemplo, que es imposible juzgar rectamente cuando se afirma y niega algo. Mas si todas las leyes lógicas son leyes del juicio y el juicio es, en última instancia, un acto de un sujeto psicológico, no por ello las leyes lógicas son leyes descriptivas de tales actos y, por tanto, meras leyes psicológicas, sino que son leyes normativas del juicio. La fundamentación de la lógica en la psicología jamás significó en Brentano la justificación empírica de las leyes lógicas, eventualmente, por un procedimiento inductivo à la Mill (BRENTANO, 1880, p. 213s.). Las leyes lógicas, aun siendo leyes del juicio, son leyes a priori inmediatamente evidentes basadas, en última instancia, en meras relaciones analíticas entre conceptos. En este carácter a priori se funda, en última instancia, su normatividad.

Si es claro que Brentano construye toda su concepción de lógica sobre el presupuesto de que el juicio es en última instancia el portador de verdad, obviamente el 
habrá de oponerse a una concepción de lógica según la cual el portador originario de verdad es la proposición y las leyes lógicas y sus principios básicos son leyes y principios que dicen respecto de la relación entre proposiciones. Tal es el caso de lógica pura que propone Husserl en "Investigaciones lógicas" y que, según él, debe ser la base de toda lógica como Kunst.

Aun cuando Husserl nunca acusa explícitamente a Brentano de psicologista en ningún momento, hay una distancia insuperable entre el platonismo de "Investigaciones lógicas" y el anti-platonismo radical de Brentano. Más aun, Brentano niega firmemente que el platonismo sea necesario para asegurar la objetividad de la lógica, afirmando que su concepción de lógica, sin objetos ideales o abstractos, asegura suficientemente la objetividad de la misma. Con tal fin, Brentano insiste en la tesis, de que las leyes lógicas, siendo leyes del juicio, son leyes del juicio de todo ser racional posible, incluso de Dios, y no meramente de la especie homo (BRENTANO, 1962, p. 153, 157).

Brentano repite una y otra vez que él nunca afirmó el subjetivismo (BRENTANO, 1971, II, p. 180; 1925, p. 147ss., 194). Pero son cosas diferentes el que no lo haya afirmado y el qué lo pueda evitar en base a los presupuestos de los cuales parte. La pregunta esencial es si se puede combatir eficazmente el psicologismo si al mismo tiempo se mantiene el MP. Esta consideración nos lleva a entender mejor la naturaleza de la fundamentación psicológica de la lógica.

Una respuesta superficial y rápida a esta cuestión, consiste en decir que el psicologismo es una consecuencia de una psicología naturalista y mecanicista y que, por tanto, como Brentano defiende una psicología descriptiva e intencional, él está libre de esta acusación (ROLLINGER, 2004). Una consideración mas detenida muestra, sin embargo, que aquí lo esencial no es la oposición causal-descriptivo, sino el hecho de que esa psicología descriptiva establece verdades a priori.

Las verdades de la psicología descriptiva, incluidas pues las verdades referentes al juicio son verdades a priori, y por tanto universales, necesarias (BRENTANO, 1982, p. 24, 73). Si, por un lado, se debe insistir en la tesis de que para Brentano, en última instancia, toda verdad debe tener un fundamento empírico, se debe, por otro, destacar 
las peculiaridades de su idea de experiencia, más próximas del empirismo aristotélico que del inglés, y en la cual se contempla la posibilidad de lo que posteriormente habrá de llamarse intuición eidética. Esta intuición eidética debe ser pensada en estrecha relación con la tesis del Begriffempirismus. Todo concepto tiene en última instancia un origen empírico. No obstante lo anterior, no todo concepto proviene de algo así como de una abstracción que sigue un modelo induccionista y, más aún, hay verdades a priori, que son verdades negativas existenciales que contienen meras relaciones entre conceptos. Las verdades referentes al juicio en cuanto tal, incluidas todas las leyes lógicas, son verdades de este tipo.

\section{Semántica y MP: la polémica de la escuela de Brentano con el realismo lógico}

Ya observamos que hay tres formas diferentes e independientes de psicologismo y anti-psicologismo y, en consecuencia, que se puede ser psicologista en una de ellas y no en otra. Aplicado lo anterior a la escuela de Brentano, resulta que su evaluación no precisa ser homogénea según todas y cada una de ellas. Aun cuando se conceda que la escuela de Brentano pueda responder exitosamente a la acusación de psicologismo en la lógica, parece inevitable conceder que sus dificultades en la semántica son bien mayores. Claramente Brentano tiende a considerar conceptos y significaciones como entidades mentales y, en este sentido al menos, puede con fundamento ser acusado de psicologista. Así como hemos distinguido tipos de psicologismo, hemos distinguido dos tipos de relativismo. Ahora bien, parece claro, que aun cuando tal vez el MP no necesariamente conduzca al relativismo genérico, no obstante, haga inevitable el relativismo individual, poco importando aquí que una psicología descriptiva sea poseedora de verdades a priori.

\section{Psicologismo y platonismo}

El problema que coloca Brentano es si es posible asegurar la objetividad de la verdad sin platonismo, o, en última instancia, de si es posible anti-psicologismo sin apelo 
ninguna a la idealidad. Que la superación del psicologismo necesariamente implica justificar alguna forma de normatividad, está fuera de duda, la cuestión es si la única forma de justificar normatividad es la postulación de objetos ideales.

Si Brentano distingue claramente dos tipos de verdades, factuales y lógicamente necesarias, en ningún momento esboza la idea de una evidencia puramente racional. Esto no es casualidad. Admitir algo así como una fuente lógica de conocimiento, como Frege, obligaría a Brentano a inclinarse a una posición similar a la de Herbart, el cual, justamente por aceptar dos fuentes de conocimiento irreductibles, tiene que combatir el MP. Mantener el MP exige aceptar que la fuente última de verdad es la percepción interna y, por tanto, remitir a esta también las verdades lógicamente necesarias. ¿Pero cómo? Si la percepción interna parece necesariamente ser, en cuanto empírica, una pura constatación de hechos, ¿cómo puede ella, entonces, ser fuente de una conciencia normativa? La única salida parece ser admitir una conciencia normativa originaria a la cual se accede ciertamente por la percepción interna, pero que, sin embargo, no se identifica ni se funda en ella. Es a esto a lo cual, en ultima instancia, tiende Brentano en su lucha contra el subjetivismo, algo que se expresa mucho mas claramente en su ética, que en su lógica.

La cuestión de la relación de Brentano al psicologismo remite, pues, en última instancia, a la noción de percepción interna y de lo que se deba entender por tal. Aun cuando los comentadores no se han centrado en ella hasta ahora, como debería acontecer, no es casualidad que la misma ocupe una y otra vez a Brentano y sus discípulos. Más aún, todo el problema del psicologismo y de la fundamentación de la objetividad en su mínima expresión termina remitiendo en Brentano a la relación entre percepción interna y principio de identidad y no contradicción. Se debe distinguir entre afirmar que, aun cuando todo concepto es empírico, existen juicios a priori, que se fundan en meras relaciones entre ellos; afirmar que las leyes de la psicología descriptiva son de este tipo; afirmar que las leyes lógicas, fundadas en leyes de la psicología descriptiva, también lo son, y afirmar, finalmente, que los principios lógicos fundamentales de identidad y no contradicción son analíticos. En cuanto las tres primeras tesis no son problemáticas, la cuarta sí lo es porque, considerada la cuestión en 
su totalidad, parece terminar conduciendo a un círculo. No todo se soluciona, pues, diciendo que (todas) las leyes lógicas son analíticas, sino que se exige una conciencia normativa originaria. Ahora bien, esta conciencia normativa, y el principio fundamental que en ella se funda, tienen que estar dados en la percepción interna y ser conocidos en base a ella (compare por ej. BRENTANO, 1880, p. 224). No por ello, sin embargo, esta conciencia normativa se reduce a la percepción interna o puede ser sin más identificada con ella. Esta parece ser la última elaboración de la posición brentaniana posible en base a los principios de los cuales hemos partido.

\section{Motivos particulares de la acusación de psicologismo a y en la escuela de Brentano}

El objeto inmanente

Una de las características peculiares del PS es que él consistió en buena medida en una permanente redefinición de los partidos en pugna, de modo tal que, a través del desarrollo de la polémica, la línea divisoria entre ambos va variando y, autores que se consideraban a sí mismos anti-psicologistas dadas las coordenadas de su período, pasan a ser considerados psicologistas por autores posteriores. En tal sentido, un caso particularmente importante lo representa Frege, pues, según su diagnóstico, es psicologista toda filosofía que acepta el principio de inmanencia (PI), o sea, la tesis cartesiano-lockeana de que el único objeto directo e inmediato de mi conocimiento son mis propias representaciones (Vorstellungen).

Sobre tal punto de vista, es comprensible que, por este motivo, haya existido la sospecha de psicologismo por parte del realismo lógico con respecto a la escuela de Brentano y, esto, no sólo porque la propia propuesta del MP surgió históricamente vinculado al PI, sino, más específicamente, porque, en algunas de sus formulaciones iniciales, la idea de intencionalidad aparece comprometida con el PI.

Aun cuando existen al día hoy controversias, los estudios más cuidadosos sobre esta cuestión muestran que, en una cierta fase de su evolución, y pese a que oscila (CRUDZIMSKI, 2001, p. 2s), Brentano tendió a la tesis que la intencionalidad se dirigía 
primariamente a un objeto inmanente, tesis ésta que el no solo abandonará posteriormente, sino que, incluso, negará jamás haber defendido.

La cuestión referente al objeto inmanente dio lugar a una dura polémica en la propia escuela de Brentano, desatada por Höfler (1890, \ 6, p. 6) y Twardowski (1982/1894, p. 3ss.), al observar que en "Psicología del punto de vista empírico" Brentano usa indiferentemente los conceptos de contenido y objeto (Inhalt y Gegenstand) y exigir la distinción de los mismos (Cfe. MEINONG, 1899, p. 185ss.). El segundo y decisivo paso en esta historia será dado por Husserl, quien, retomando la tesis de Twardowski, exigirá ulteriores distinciones en la misma, tales como la existente entre el contenido real e ideal (HUSSERL, 1979, p. 349ss.). Con esto se delinean claramente dos líneas de desarrollo de la idea de intencionalidad, siendo que una va en la dirección de una complicación por el lado del acto (Husserl), en tanto que la otra de una complicación por el lado del objeto (Meinong), ambas, en el fondo, intentando dar cuenta del hecho básico de que si la vivencia intencional requiere necesariamente la existencia de un objeto, ella, sin embargo, no siempre se dirige a un objeto real o existente.

\section{La concepción epistémica de verdad}

En la concepción brentaniana de verdad es dable diferenciar entre una teoría correspondencial (1962, p. 23) inicial y una teoría puramente epistémica posterior, que identifica verdad con evidencia. Si admitimos una teoría correspondencial, precisamos de algo que asegure la verdad tanto del juicio afirmativo verdadero como del juicio negativo y, como ese algo no puede ser el objeto real, ya que en el caso de los juicos verdaderos negativos este simplemente no existe, el mantenimiento de la teoría exige la introducción de entidades no reales tales como el "ser" y "no-ser" de la cosa. La teoría correspondencial está, pues, vinculada a la aceptación de ciertos Irrealia y, por tanto, será abandonada junto con el abandono de los Irrealia en general en la fase reista.

Marty mantiene la teoría original de Brentano con respecto a la verdad, incluso después que éste la abandona. Esto da origen a una polémica entre maestro y discípulo que habrá de extenderse por años (BRENTANO, 1971, II, p. 172; 1925, p. 194; 1966, 
p. 201, 214, 298). Marty acompaña parcialmente a Brentano en su abandono de los Irrealia, eliminando por ejemplo el objeto inmanente. No obstante, Marty no acompaña a Brentano en la teoría de los modos de representación (esto es, de que un objeto puede ser representado de diferentes formas), la cual permitía a éste eliminar todos los Irrealia, siendo obligado por ello a mantener Irrealia tales como relaciones y, lo que nos interesa destacar ahora, contenidos judicativos (Urteilsinhalte). Los Urteilsinhalte de Marty no son pensamientos (Gedanken) o sentido de enunciados, pues ellos no son portadores de verdad (Wabrheitsträger), sino aquello que funda la verdad del juicio (Wabrheitsmacher). El punto esencial de la discusión en torno a la necesidad o no de Urteislinhalten entre Brentano y Marty es, en definitiva, el si es necesario o no admitir la existencia de algo transcendente a la subjetividad, en este caso, al acto de juzgar, para garantir la objetividad. Para Marty una concepción puramente epistémica de verdad condena al subjetivismo, para Brentano no (MARTY, 1916, 155ss.).

Si Marty, con la postulación de Urteilsinablten va a oponerse a Brentano, por un lado, por otro, va a oponerse también a Stumpf, para quien, el estado de cosas (Sachverhalt) es un contenido inmanente a la conciencia (MARTY, 1908, p. 391-392, 400). Lo que acontece en el caso de la noción de Urteilsinhalt, es una constante en las relaciones entre Stumpf y Marty, siendo Marty la variante más transcendentalista y Stumpf la más inmanentista dentro de la escuela de Brentano.

\section{MP y Apsicologismo}

Si es esencial a la autoidentidad de la escuela de Brentano el mantenimiento del MP, justamente por ello, su disolución estará vinculada al abandono de ese método, sea en la dirección de la teoría del objeto (Gegenstandstheorie), sea en la dirección de la fenomenología. Consideremos este proceso desde la primera y menos conocida perspectiva. Meinong se ve impulsado a la tesis de que son necesarias dos ciencias básicas de la filosofía, a saber, la psicología y la Gegenstandstheorie (MEINONG, 1921, p. 5-11; 1904, p. 23ss.). Con esta tesis, Meinong abandona decisivamente el MP y, con él, la escuela de Brentano, pues la propia idea de Gegenstandstheorie implica una doctrina de 
objetos que, sin eliminar la cuestión del acceso a los mismos, defiende la idea de que es posible una consideración esencialmente a-psicológica de tales objetos, esto es, libre de toda perspectiva subjetiva (HÖFLER, 1906, p. 216, 219). Así, de este modo, el "objetivismo", por usar la expresión de sus críticos, termina concibiéndose a si mismo como la necesaria consecuencia del anti-psicologismo.

Si Marty, y a través de su teoría de los Urteilsinhalte como Wahrgeisträger necesarios para superar todo subjetivismo, se revela como el brentaniano más inclinado a integrar dentro del horizonte del MP la idea de transcendencia y, en tal sentido, el más receptivo a las solicitaciones del anti-psicologismo característico del realismo lógico, sin embargo, él es también el único brentaniano que establece una discusión metódica radical con el realismo lógico en el sentido de cuestionar duramente la posibilidad de una consideración puramente objetiva o a-psicológica de objetos, incluso, pues, de objetos que son pensados como siendo esencialmente transcendentes (MARTY, 1908, p. 304, 307).

Si por su defensa de la transcendencia Marty plantea una polémica dentro de la escuela con el propio Mestre, por su defensa radical del MP frente a objetivismo y consideración apsicológica, Marty establece una nueva etapa en la historia del PS, etapa poco conocida y aun menos estudiada.

\section{Conclusión}

La participación de la escuela de Brentano en el PS no se deja reducir a un único capitulo, siendo variada tanto desde el punto de vista sistemático como histórico, componiéndose de diferentes momentos que tienen un cierto grado de independencia. Si no cabe duda de que la escuela de Brentano como un todo defendió el MP, y por tal motivo fue acusada de psicologismo, no puede caber duda, tampoco, que ella resistió desde siempre tal acusación y elevó la pretensión de haber desenvuelto un antipsicologismo peculiar, libre tanto de platonismo cuanto de idealismo y subjetividad transcendental. Reconocer lo anterior, sin embargo, no implica conceder sin más que, en todos los planos y sobre todos los puntos de vista, su alternativa sea satisfactoria, mismo 
porque, más allá de la adhesión al MP, existen diferencias importantes entre sus integrantes.

\section{Bibliografia}

ADICKES, E. Rezension Anton Marty: Was ist Philosophie? Deutsche Literatur-Zeitung, Nr. 22, S. 855-857, Jahr. 1899.

BRENTANO, F. Die Habilitationsthesen. In: Über die Zukunft der Philosophie. Hamburg: Meiner, 2013.

BRENTANO, F. Psychologie vom empirischen Standpunkt. 1 Bde. Hamburg: Felix Meiner, 1971.

BRENTANO, F. Psychologie vom empirischen Standpunkt. 2 Bde. Hamburg: Felix Meiner, 1971.

BRENTANO, F. Über die Zukunft der Philosophie. Wien: Alfred Hölder, 1893.

BRENTANO, F. Versuch über die Erkenntnis aus dem Nachlass hrsg. v. A. Kastil. Leipzig, Meiner, 1925; erweitert u neu eingeleitet von F. Meyer Hillebrand. Hamburg, Meiner, 2, 1970.

BRENTANO, F. Wabrheit und Evidenz. Erkenntnistheoretische Abhandlungen und Briefe. Ausgewählt, erläutert u. eingeleitet v. O. Kraus. Leipzig, Meiner, 1940, Hamburg, Meiner, 1962.

BRENTANO, F. Die Lehre vom richtigen Urteil mit Vorwort u. Anmerkungen hrsg. von F. MayerHillebrand. Bern, Francke, 1956.

BRENTANO, F. Die Abkehr vom Nichtrealen. Mit Einleitung u Anmerkungen hrsg. von F. Mayer Hillebrands. Bern, Francke, 1966.

BRENTANO, F. Deskriptive Psychologie. hrsg. V R. M. Chisholm u W. Baumgartner. Hamburg, Meiner, 1982.

BRENTANO, F. E. L. Logik. Das Manuskript der Logik Vorlesung aus der zweiten Hälfte der achtziger Jahre, 1880. Disponível em: <http://gams.uni-graz.at/archive/objects/o:bag.el.80html-norm/methods/sdef:HTML/get>.

CAVALLIN, J. Content and object. Husserl, Twardowski Psychologism Dordrecht. Boston/ London: Kluwer, 1997.

CERNOCH, G. W. Der Subjektivismus bei Franz Brentano in den Grenzen von Psychologie und Logik (Über den Ursprung der sittlichen Erkenntnis). Disponível em: $<$ https://philarchive.org/archive/CERDSB $>$.

CRUDZIMSKI, A. Intentionalitätstheorie beim frühen Brentano. Dordrecht: Springer, 2001. 
ESCHENMEYER, J. Die Psychologie und ihre zentrale Stellung in der Philosophie. Eine Einführung in die wissenschaftliche Philosophie. Halle: Max Niemeyer, 1914.

FRECHETTE, G. Kant, Brentano e Stumpf sobre psicología e anti-psicologismo. Peri, v. 8, n. 1. p. 1-11, 2016.

FRIES, J. F. Neue oder anthropologische Kritik der Vernunft. Berlin: Verlag Öffentliches Leben, 3. Bde, 1935.

HEIDEGGER, M. Die Lehre vom Urteil im Psychologismus. In: HEIDEGGER, M.: Frühe Schriften. Frankfurt am Main: Vittorio Klostermann, 1972.

HÖFLER, A. Logik Vienna. Temspki Verlag, 1890.

HÖFLER, A. Sind wir Psychologisten? In: Atti del V Convegno internazionale de Psicologia. Roma Forzan, 1906.

HÖFLER, A. Anæeige von Meinong Über Annahmen. Göttingsche Gelehrte Anzeigen 3 (Göttingen 1906), 1906. p. 209-227.

HUEMER, W. Husserl's critique of Psychologism and his relation to the Brentano school. In: CHRUDZIMSKI, A. Y HUEMER, W. (eds.) Phänomenology and analysis. Essays on central european philosophy. Frankfurt: Ontos, 2003, p. 199-214.

HUSSERL, E. Briefwechsel Brentano Schule. Husserliana Dokumente, III/1Briefe, 1994.

HUSSERL, E. Besprechung von K. Twardowski. In: HUSSERL, E. Aufsätze und Rezensionen (1890-1910). Mit ergänzenden Texten hsg. von Bernard Rang. Husserliana, XXIII, Den Haag, Nijhoff. 1979. (Hua, XXII).

HUSSERL, E. Logische Untersuchungen. Erster Band. Prolegomena zur reinen Logik. Ed. Elmar Holenstein. Husserliana XVIII. The Hague: Martinus Nijhoff, 1975. (Hua, XVII).

HUSSERL, E. Zweiter Band Untersuchungen zur Phänomenologie und Theorie der Erkenntnis. Ed. Ursula Panzer. Husserliana XIX, I. The Hague, Boston, Lancaster: Martinus Nijhoff, 1984

HUSSERL, E. Zweiter Teil Untersuchungen zur Phänomenologie und Theorie der Erkenntnis Ed. Ursula Panzer. Husserliana, XIX, II. The Hague, Boston, Lancaster: Martinus Nijhoff, 1984.

HUSSERL, E. Referat. Anton Marty Untersuchung zur Grundlegung der allgemeinen Grammatik. Deutsche Literaturzeitung, n. 18, p. 1106-1110, 1910.

KAMITZ, R. Die Rolle der deskriptiven Psychologie in der Logik Franz Brentano. Brentano Studien, 2, p. 79-90, 1989.

KASTIL, A. Jakob Friedrich Fries Lehre von der unmittelbaren Erkenntnis. Eine Nachprüfung seiner Reform der theoretischen Philosophie Kants. Göttingen: Vandenhoeck and Ruprecht, 1812. (Abhandlungen der Fries'schen Schule, NF, IV Band. 1. Heft). 
KASTIL, A. Brentano und der Psychologismus. Zeitschrift für philosophische Forschung. Bd. 12, H. 3, p. 351-359, 1958.

KRAUS, O. Einleitung. In: BRENTANO, F. Psychologie vom empirischen Standpunkt. 2 Bde. Hamburg: Felix Meiner, 1971. p. XVI-XCIIII.

KRAUS, O. Besprechung Cohen. Deutsche Literaturzeitung, 1929. Heft 30.

KUSCH, M. Psychologism. A case Study in the Sociology of Philosophical Knowledge. London: Routledge, 1995.

MARTY, A. Was ist Philosophie? [Inaugurationsrede gehalten beim Antritt des Rektorates der k. k. deutschen Karl-Ferdinands Universität zu Prag am 16. November 1896].

MARTY, A. Über subjektlose Sätze und das Verhältnis der Grammatik zu Logik und Psychologie. Erster bis dritter Artikel 1884; Viertes bis siebenter 1894-1895.

MARTY, A. Untersuchungen zur Grundlegung der allgemeinen Grammatik und Sprachphilosophie. Halle: a.s. Niemeyer, 1908.

MARTY, A. Vorlesungen zur deskriptiven Psychologie. Hrsg. M. Antonelli und J.Ch. Marek. Amsterdam-Atlanta: Rodopi, 2011.

MARTY, A. Einleitung zu Anton Martys Elemente der deskriptiven Psychologie. Johann Christian Marek y Barry Smith. Conceptus XXI, N. 53-54, S. 33-47, 1987.

MARTY, A. Raum und Zeit. Ed. J. EISENMEIER, A. KASTIL, O. KRAUS. Halle: Max Nyemeier, 1916.

MEINONG, A. Über philosophische Wissenschaft und ibre Propädeutik. Wien: Alfred Hölder, 1885. (GA V, s. 1-196).

MEINONG, A. Über Gegenstände höherer Ordnung und deren Verhältnis zur inneren Wahrnehmung. Zeitschrift für Psychologie und Physiologie der Sinnesorgane, 21, 1899, p. 182-272.

MEINONG, A. Über Gegenstandstheorie. In: Untersuchungen zur Gegenstandstheorie und Psychologie. Leipzig: Johanes Ambrosius Barth, 1904. p. 1-50.

MEINONG, A. Über Annahmen 1902. Leipzig: Johanes Ambrosius Barth. Ergänzungsband 2 der Zeitschrift für Psychologie und Physiologie der Sinnesorgane. 1902.

MEINONG, A. Über die Stellung der Gegenstandstheorie im System der Wissenschaften. Zeitschrift für Philosophie und philosophische Kritik, 129, 1906, p. 38-94 1. Artikel; p. 145-207 2te. Artikel; 130, 1907, p. 1-46 3. Artikel.

MEINONG, A. Selbstdarstellung in Die deutsche Philosophie der Gegenwart in Selbstdarstellung. Band I. Leipzig, Meiner, 1921. p. 91-150. 
MEINONG, A. Für die Psychologie und gegen den Psychologismus in der allgemeinen Werththeorie. Internationale Zeitschrift für Philosophie der Kultur, Tübingen, 1912. Bd. 3.

MODENATO, F. Meinong's Theory of objects. An attempt at overcoming psychologism. Grazer Philosophische Studien, 50, p. 87-112, 1995.

ROLLINGER, R. D. Brentano and Husserl. In: JACQUETTE, D. (ed) The Cambridge companion to Brentano. Cambridge University Press, 2004. p. 255-276.

ROLLINGER, R. D. Husserls Position in the school of Brentano. Dordrecht: Kluwer, 1999.

SIMONS, P. Brentano's reform of logic. Topoi, 6, 1987, pp. 25-38 Austrian philosophers on truth. Textor, Mark (ed.). Austrian contribution to analytic Philosophie. London: Routledge, 2014.

STUMPF, C. Psychologie und Erkenntnistheorie. München: Verlag der K. Akademie, 1892.

STUMPF, C. Erkenntnislehre. Langerich, Pabst Science Publishers, 2011.

STUMPF, C. Selbstdarstellung. R. SCHMIDT, Die Philosophie der Gegenwart in Selbstdarstellungen. 5. Band (s. 204-265) Leipzig: Meiner, 1924.

STUMPF, C. Die empirische Psychologie der Gegenwart. Im neuen Reich, v. 4, n. 2, p. 201-226. 1874.

STUMPF, C. Zur Einteilung der Wissenschaften. Berlin, Königl. Akademie der Wissenschaften, 1907.

TWARDOWSKI, K. Zur Lehre von Inhalt und Gegenstand der Vorstellung. Eine psychologische Untersuchung. München: Philosophia Verlag / A. Hölder, 1982/1894.

TWARDOWSKI, K. Psychology, Vs. Physiology and Philosophy; In: On actions, products and other topics in Philosophie. Amsterdam: Rodopi, 1999. (Ed. Por Johannes L. Brandl and Jan Wolenski. p. 41-64).

TWARDOWSKI, K. Gesammelte deutsche Werke. Eds. Brozék, A.; Jadacki, J.; Stadler, F. Berlin: Springer, 2015. (Institut für den Wiener Kreis).

RECEBIDO: 03/06/2019

APROVADO: 07/06/2019
RECEIVED: 06/03/2019

APPROVED: 06/07/2019 